\title{
Parietal updating of limb posture: An event-related fMRI study
}

\author{
Asa Pellijeff ${ }^{\mathrm{a}}$, Leonardo Bonilha ${ }^{\mathrm{a}}$, Paul S. Morgan ${ }^{\mathrm{b}}$, \\ Kirsten McKenzie ${ }^{\text {a }}$, Stephen R. Jackson ${ }^{\mathrm{a}, *}$ \\ a School of Psychology, University of Nottingham, Nottingham, UK \\ ${ }^{\mathrm{b}}$ Division of Academic Radiology, School of Medical and Surgical Sciences, University of Nottingham, Nottingham, UK
}

Received 27 October 2005; received in revised form 9 January 2006; accepted 20 January 2006

\begin{abstract}
The posterior parietal cortex (PPC) is thought to integrate different kinds of sensory information (e.g., visual, auditory, somatosensory) to produce multiple representations of space that are each associated with different types or combinations of action; such as saccadic eye movements and reaching or grasping movements of the upper limb. Lesion studies in monkeys and in humans have shown that reaching movements to visually defined and to posturally defined targets can be dissociated from one another; indicating that different regions of the parietal cortex may code the same movement in either extrinsic (visual) or intrinsic (postural) coordinates. These studies also suggest that regions within the posterior parietal cortex play an important role in maintaining an accurate and up-to-date representation of the current postural state of the body (the body schema). We used event-related functional magnetic resonance imaging (fMRI) to investigate those brain areas involved in maintaining and updating postural (i.e., non-visual) representations of the upper limb that participate in the accurate control of reaching movements. We show that a change in the posture of the upper-limb is associated with a significant increase in BOLD activation in only one brain region-the superior parietal cortex, particularly the medial aspect (precuneus). We note that this finding is consistent with the suggestion, based upon human neurological investigations and monkey electrophysiology, that this region of the PPC may participate in the dynamic representation of the body schema, and is the most likely location for damage leading to errors in visually guided reaching to non-foveated target locations. We also note that this brain area corresponds to a region of PPC recently identified as the human homologue of the Parietal Reach Region (PRR) observed in the monkey brain that has been thought to represent reaching movements in eye-centred coordinates.
\end{abstract}

(C) 2006 Elsevier Ltd. All rights reserved.

Keywords: Parietal cortex; Reaching; Frame-of-reference; Intrinsic coordinates; Parietal reach region; fMRI

\section{Introduction}

The posterior parietal cortex (PPC) is thought to play a key role in the representation of corporeal and peripersonal space, and in the sensorimotor transformations associated with goaldirected movements (for reviews see Andersen, Snyder, Bradley, \& Xing, 1997; Rizzolatti, Fogassi, \& Gallese, 1997; Snyder, 2000). In humans damage to the PPC leads to disorders in the representation of space (e.g., hemispatial neglect) and impairments in the planning and control goal-directed movements (e.g., ideomotor limb apraxia and optic ataxia) (Balint, 1909; Critchley, 1953; De Renzi, 1982; Jackson, Newport, Mort, \&

\footnotetext{
* Corresponding author at: School of Psychology, The University of Nottingham, Nottingham NG7 2RD, UK.

E-mail address: stephen.jackson@ nottingham.ac.uk (S.R. Jackson).
}

Husain, 2005; Liepmann \& Maas, 1907; Sirigu et al., 1996). Understanding the nature of the sensorimotor transformations carried out by human PPC is a fundamental problem for neuroscience and of considerable clinical importance in treating the consequences of stroke, and other neurological disorders that affect this brain region.

To execute goal-directed movements, such as reaching to pick up an object, information specified in extrinsic (spatial) coordinates must be transformed into a motor plan that can be expressed within intrinsic (motor) coordinates. For reaching movements directed to visually defined targets, this will involve translating visual information that is coded initially in retinotopic coordinates, into a motor plan that specifies the sequence of postural changes required to bring the hand to the target. Electrophysiological and lesion studies in the monkey indicate that there is no single, supramodal, map of space that is used to guide movements. Instead, movements appear to be 
capable of being planned and controlled within multiple coordinate systems, each one attached to a different body part (see Buneo \& Andersen, in press; Gross \& Graziano, 1995). Thus, recordings in the superior parietal lobule (SPL) of the monkey brain have demonstrated the existence of a 'parietal reach region' (PRR) in which the targets of reaching movements are represented within eye-centred rather than body-centred coordinates (Batista, Buneo, Snyder, \& Andersen, 1999). By contrast, other regions of $\mathrm{PPC}$, such as area 5 or area $7 \mathrm{~b}$, have few visual inputs, are strongly interconnected with somatosensory and motor cortices, are dominated by somatic and motor responses, and most likely represent limb position in intrinsic (postural) coordinates (Rushworth, Nixon, \& Passingham, 1997; Rushworth, Johansen-Berg, \&Young, 1998; Sirigu et al., 1995).

Rushworth et al. (1997) demonstrated that bilateral lesions to different regions of the monkey PPC produce doubly dissociable effects on reaching movements. Specifically, lesions to areas PG and PFG (including the lateral intraparietal region (LIP - the so-called parietal eye field) and the PRR) impair visually guided reaching in the light but do not affect reaching movements made in the dark to target positions that are defined posturally. However, the reverse is true for bilateral lesions of areas PE and $\mathrm{PF}$ (area 7b, area 5, and the medial intraparietal region), which severely impair reaching movements in the dark to posturally defined targets, but leave unaffected reaching movements made in the light to targets that are defined visually. These data indicate that reaching movements may be coded in extrinsic and intrinsic coordinates in different regions of the monkey PPC. Furthermore in a companion study, Rushworth and colleagues (Rushworth et al., 1998) demonstrated that while lesions to area $7 \mathrm{~b}$, area 5 , and the medial intraparietal region do not appear to disrupt the transformation of target information from retinal coordinates into body-centred (shoulder) coordinates, they do impair the transformation between desired hand position in extrinsic coordinates and the postural configuration of the arm (intrinsic coordinates).

It remains to be clearly demonstrated whether the functional organisation of the monkey parietal lobes (investigated largely using electrophysiological recording techniques) is replicated in the human PPC (investigated primarily using neuropsychological and functional brain imaging approaches - see other papers in this volume for a discussion of this topic). And we have speculated that the bilateral organisation of neural systems coding movements in intrinsic (postural) and extrinsic (eye-centred) coordinates observed in the monkey, may be differently organised in the human PPC. Specifically, we consider that while the human right PPC may be predisposed to plan and control movements in eye-centred coordinates, the human left PPC is biased to plan and control movements in postural coordinates (note that there are strong interhemispheric connections in the undamaged human brain that would allow these two systems to interact (Gazzaniga, 2000)).

The above suggestion is consistent with the finding that optic ataxia - misreaching under visual guidance - is quite different following unilateral left and right hemisphere damage De Renzi (1996). Thus, while unilateral damage to the right PPC typically leads to so-called 'field' effects, in which one or both limbs misreach to targets presented in the left visual field alone, unilateral lesions of the left PPC most often produces 'hand' effects, where only the contralateral limb misreaches when moving to targets presented in either visual hemifield (Perenin \& Vighetto, 1988; Ratcliffe \& Davies-Jones, 1972). It is also consistent with the finding that left PPC damage produces deficits in postural representation (Sirigu et al., 1995) whereas right PPC damage gives rise to impaired reaching movements to targets that are defined visually, but leaves unaffected reaching movements made, without vision, to targets that are defined posturally (Jackson, Newport, Husain, Harvey, \& Hindle, 2000).

Irrespective of the whether the spatial representations used to guide reaching movements are more strongly lateralised in the human PPC than in the monkey, recent neuropsychological evidence clearly indicates that in humans the left superior parietal lobule at least may play a key role in actively maintaining an accurate and up-to-date representation of the current postural state of the body (Wolpert, Goodbody, \& Husain, 1998). In this study we sought converging evidence for this view by using functional brain imaging techniques to study movements directed to posturally defined target locations in the absence of vision. Specifically, we used event-related functional magnetic resonance imaging (fMRI) to investigate which brain areas are specifically involved in maintaining and updating the postural (i.e., non-visual) representations of the upper limb that participate in the accurate control of reaching movements.

\section{Methods}

\subsection{Participants}

Event-related fMRI was carried out on thirteen neurologically-healthy adult volunteers (eight females; mean age 24.6 years). Twelve of the participants reported that they were right-handed and one claimed to be left-handed. Note that the BOLD activations for the left-handed subject were not qualitatively different from those observed for subjects reporting themselves to be right handed. All subjects showed a similar pattern of activation, which was visually assessed by the researchers before data was submitted to random-effects analysis. All participants gave informed consent to take part in the study. Ethical approval for this study had been obtained from the University of Nottingham Ethics Committee.

\subsection{Image acquisition}

Imaging was performed at the Queen's Medical Centre, using a Philips $1.5 \mathrm{~T}$ scanner equipped with a multiple-element 'sense' head-coil. We obtained 32 contiguous axial slices $(20.8 \mathrm{~cm}$ FOV, $64 \times 64$ matrix, $3 \mathrm{~mm}$ slice thickness $)$ parallel to the AC-PC plane, which covered the whole brain using a gradientrecalled $\mathrm{EPI}$ sequence $(\mathrm{TR}=3.5 \mathrm{~s})$.

\subsection{Experimental design}

Participants rested comfortably in a supine position in the MR scanner. Their extended arms were oriented in a relaxed position parallel to the trunk and they had their eyes closed throughout both experiments. Participants wore ear protection while they were in the scanner, but also wore a set of headphones and received auditory instructions from the experimenter via an intercom system. Participants completed two different experimental tasks; a chin pointing experiment and a thumb-pointing task. 


\subsubsection{Experiment 1: chin pointing task}

On each trial participants were required to execute a reaching movement (point-to-point) from a start position at waist level so as to bring their index finger to contact the point of the chin. The starting position (posture) of each movement was varied across trials. All participants were asked to place one hand (left or right) on their upper body and keep the other limb immobile by their side. All participants started with the right hand on their upper body (moving hand) and the left hand (non-moving) at their side. Their task on each trial was to make a pointing movement from a position on their torso to the point of their chin, hold this posture for several seconds, and then return their hand to its starting position. Movements were precisely paced by providing subjects with auditory instructions. After three trials had originated from one start location, participants were instructed to change the position of their moving hand to a new start location (i.e., a position on their upper body that was significantly different from the previous used start location). Having moved to a novel start position, participants completed three pointing movements from this location before selecting another start location. Pilot studies were conducted outside the scanner to examine movement durations. These suggested that novel and repeated movements had comparable durations and would have been unlikely to vary significantly during the experiment and would not influence the hemodynamic response function that is modelled with somewhat poor temporal resolution by the fMRI. At the start of each trial participants were presented with an auditory cue, 'Start', instructing them to start the movement (lasting $1 \mathrm{~s}$ ), at which point they moved to the target location. Participants had an average of $6 \mathrm{~s}$ to perform this movement (stimuli were jittered between 3.5 and $12.5 \mathrm{~s}$, within a normal distribution skewed to shorter times in order to maximize the power of the convolution of the gamma function with the data (Mechelli, Henson, Price, \& Friston, 2003) and then they heard 'Back' (lasting $1 \mathrm{~s}$ ) which instructed them to move their moving hand back to the starting position. Participants had, on average, $2 \mathrm{~s}$ (jittered between 1.5 and $2.5 \mathrm{~s}$, within a non-skewed normal distribution) to move the hand back to the starting position, after they heard 'Back'. After the three trials at one location they heard 'Move Position' (lasting 2s), indicating that they should move the position of their moving hand to a different location on the upper body. Trials were blocked for each subject in an ABBA design. Participants first completed eight sets of three sequential movements before they heard 'Please rest for $20 \mathrm{~s}$. After this rest period they heard 'Change Hand' (lasting $2 \mathrm{~s}$ ) indicating they should swap their hands around, i.e. the moving hand now became the non-moving hand and vice versa. Participants then performed 16 sets of three trials with that hand before taking another rest period and completing a further eight sets of trials with the original hand. There were 32 trial sets in total (one set $=$ three movements from one location). The experiment took around $20 \mathrm{~min}$.

\subsubsection{Experiment 2: the thumb pointing task}

The participants and scanning details for this experiment were identical to those outlined above. The experimental procedures were also very similar with one small change. Instead of reaching to a fixed target position (the chin) from a variable starting position (posture), participants were required to reach from a fixed start position to a target position that was defined posturally, and which varied across the experiment as follows. Participants were asked to place one hand (left or right) on their upper body, and keep the other limb at their side. Their task was to make pointing movements using the index finger of the hand that rested parallel to the body (moving hand) so as to touch the thumb of the hand that rested on their upper body (target hand). Participants changed the position of the target hand on their upper body when they heard 'Move Position' in an identical manner to that described above. All participants started with the right hand on their upper body and the left hand on their side. Again, they were required to make pointing movements to the target thumb three times to each target position and 'Move Position' indicated they should move their target hand to a different position on their chest.

\subsection{Data analyses}

Images were transformed into ANALYZE format using the MRIcro software (http://www.mricro.com/). Analyses of the fMRI data involved image pre-processing and statistical analysis, and was carried out using the FEAT tool (http://www.fmrib.ox.ac.uk/fsl). Images were first corrected for differences in the acquisition time between slices using Fourier-space time-series phase- shifting. Subsequently, images were submitted to motion correction (Jenkinson, Bannister, Brady, \& Smith, 2002) and to removal of non-brain regions (Woolrich, Ripley, Brady, \& Smith, 2001). Finally, the functional images were spatially smoothed by a Gaussian kernel of full-width half-maximum (FWHM) equal to $6 \mathrm{~mm}$; all volumes were normalized for mean-based intensity by the same factor; and submitted to highpass temporal filtering (Gaussian-weighted LSF straight line fitting with sigma $=64 \mathrm{~s}$ ). Time-series statistical analysis employed general linear modelling (GLM) and a Gamma function (Woolrich et al., 2001), convolving event types such as the first movement, second and third movements, rest and moving back to the original position. Contrast images, representing the differences in parameter estimates of the height of the canonical responses for modelled event types, were defined for a within subject comparison of each type of trial. Contrasts were defined to compare between the first movement and the consecutive two movements, and were weighted so both conditions (first, second and third movements combined) would have similar influence. Higherlevel analyses were carried out using FLAME (FMRIB Local Analysis of Mixed Effects) (Beckmann, Jenkinson, \& Smith, 2003). Z (Gaussianised $T / F$ ) statistic images were generated using a cluster threshold of $Z>2.3$ and a (corrected) cluster significance threshold of $P=0.05$ (Worsley, Evans, Marrett, \& Neelin, 1992.

\section{Results}

\subsection{Experiment 1}

A key issue to investigate in this experiment was to determine whether the first movement in each set of trials, i.e., that involving a novel limb posture, would elicit increased activation, compared to movements that involved repeating a postural sequence that has been used on a previous trial or trials (e.g., the second and third movements in each set). As noted by Rushworth et al. (1998), the relation between desired hand position and an associated postural configuration is considered to be less complex for movements made without visual guidance compared to movements directed to visual targets. Specifically, Rushworth et al. assume that going from a postural representation of limb position to a corresponding position in visual space requires additional processing steps that are unnecessary when moving without vision to posturally defined targets. We therefore contrasted the first movement within each set (novel posture) with the second and third movements combined (repeated posture). Note, however, that each movement was in fact selected from the continuous stream of movements that were executed within an entire block of trials.

Data are presented in Fig. 1 and in tabular form in Table 1. Analyses of these data confirmed that there was a significant increase in BOLD activation for novel posture trials compared to repeated posture trials, but this was only apparent within the superior parietal lobe bilaterally (Fig. 1). When the converse statistical comparison was carried out i.e., second/third trials (repeated posture) - first trial (novel posture) we observed no regions of increased BOLD activation $(p>0.05)$. We note that this area of activation corresponds closely to the region identified recently by Connolly, Andersen and Goodale (2003), using fMRI, as the human homologue of the monkey PRR. This will be discussed below.

\subsection{Experiment 2}

The region of increased activation in the superior parietal cortex associated with directing movements from a novel start- 


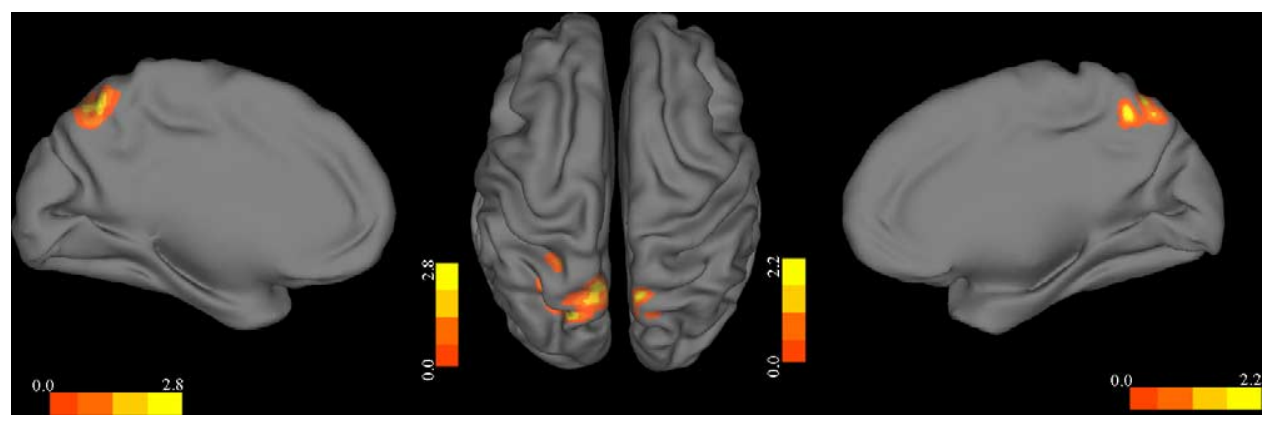

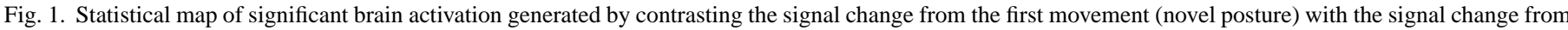

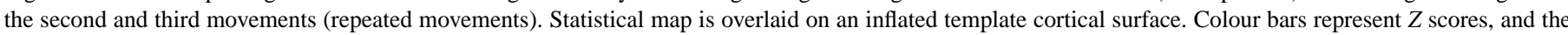

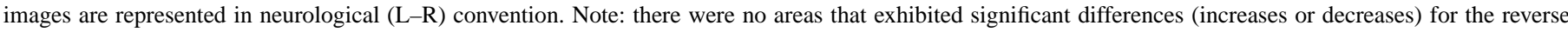
comparison (i.e., second and third movement - first movement).

Table 1

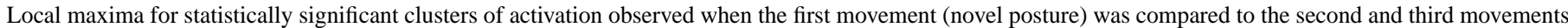

\begin{tabular}{|c|c|c|c|c|c|c|c|}
\hline \multicolumn{5}{|c|}{ Talairach coordinates } & \multicolumn{3}{|c|}{ Anatomical location } \\
\hline$X$ coordinate & $Y$ coordinate & $Z$ coordinate & Cluster & $Z$ score & Lobe & Location & Adjacent Brodmann area \\
\hline-8 & -62 & 56 & 1 & 3.85 & Parietal Lobe & Superior parietal lobule & Brodmann area 7 \\
\hline-16 & -70 & 48 & 1 & 3.32 & Parietal Lobe & Precuneus & Brodmann area 7 \\
\hline-24 & -54 & 30 & 1 & 3.32 & Parietal Lobe & Precuneus & Brodmann area 7 \\
\hline-22 & -50 & 48 & 1 & 3.05 & Parietal Lobe & Precuneus & Brodmann area 7 \\
\hline-26 & -56 & 40 & 1 & 3.04 & Parietal Lobe & Superior parietal lobule & Brodmann area 7 \\
\hline-18 & -62 & 52 & 1 & 3 & Parietal Lobe & Superior parietal lobule & Brodmann area 7 \\
\hline
\end{tabular}

ing posture that was observed in Experiment 1 conforms closely to the cortical area identified using electrophysiological recording techniques in the monkey as the likely locus of misreaching deficits in man (Battaglia-Mayer \& Caminiti, 2002; see also Battaglia-Mayer, Archambault, \& Caminiti, in press). It also supports the contention, based upon neuropsychological evidence, that regions of the superior parietal cortex may play a key role in actively maintaining up-to-date representation of the current postural state of the body (Wolpert et al., 1998; see also Head \& Holmes, 1911-1912; Critchley, 1953).

Based upon the results of Experiment 1 we created a bilateral region-of-interest (ROI) corresponding to the superior parietal region identified within Experiment 1 as associated with increased BOLD activation, and directly tested the contrast examined in Experiment 1. Specifically, we compared activation within this ROI for the first movement in each trial set (novel target posture) against the second/third movements (directed to a repeated posturally defined target location). This analysis confirmed that there was a statistically significant increase in activation within this ROI for the first versus second/third movements $(t=2.17$, corrected $p<0.025)$. This finding confirms that obtained in Experiment 1. Mean percent signal change values for the novel versus repeated posture comparison, for each hemisphere, and for both experiments are presented within Fig. 2. This figure is based upon an additional ROI analysis in which we directly compared changes in superior parietal activation using ROIs taken from published anatomical libraries. Specifically, we used ROIs based upon a manual parcellation of a single brain (colin27) in MNI space (Tzourio-Mazoyer et al., 2002) to investigate the percent of signal change within the supe-

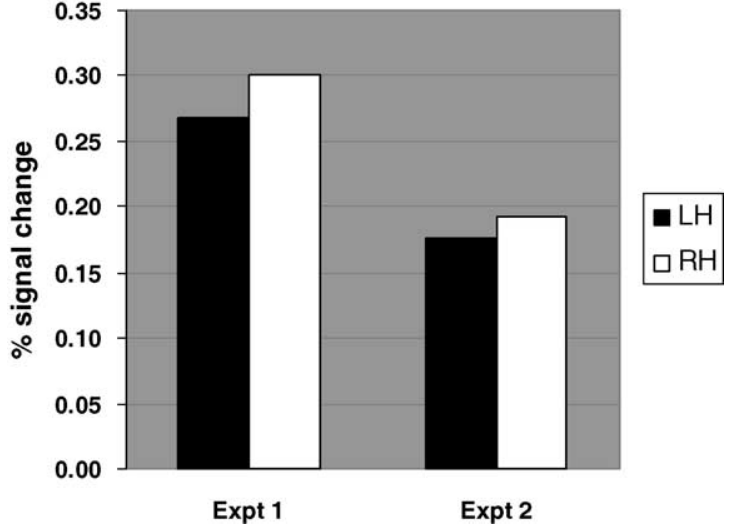

Fig. 2. Bar graph demonstrating the difference (percent of signal change) between the first movement and the following two movements for Experiment 1 the chin pointing study, and Experiment 2 the thumb pointing study. Signal change is shown for each hemisphere separately.

rior parietal lobules in the datasets obtained in Experiments 1 and 2.

\section{Discussion}

We used event-related functional magnetic resonance imaging (fMRI) to investigate brain areas involved in maintaining and updating postural representations of the upper limb that may participate in the accurate control of reaching movements. In Experiment 1 we investigated reaching movements made without vision from variable, posturally defined, starting positions to a fixed, posturally defined, target location (the point of the 
chin). In Experiment 2 we investigated reaching movements executed without vision from a fixed, posturally defined, start position to variable, posturally defined, target locations (defined by the thumb of the non-moving limb). In both experiments we compared activations associated with reaches involving a novel postural configuration of the limb, against reaches involving the repetition of a recently used limb configuration. Importantly, we show that this contrast is associated with an increase in activation in only one brain area, the superior parietal cortex bilaterally, in particular its medial aspect (the precuneus). We conclude that this region may play a key role in maintaining a continuously updated representation that codes the current configuration of the body and its parts, specified in intrinsic (postural) coordinates. This representation would conform to the notion of a body schema as proposed by Head and Holmes (1911-1912) and Critchley (1953).

The above suggestion is consistent with electrophysiological studies in non-human primates that indicate that the superior parietal lobule integrates inputs from primary somatosensory cortex to create complex representations of body posture (e.g., Sakata, Takaoka, Kawarasaki, \& Shibutani, 1973), and with neuropsychological evidence linking the superior parietal lobule to the active maintenance of a dynamic representation of the postural configuration of the body (Wolpert et al., 1998).

Damage to the superior parietal cortex has long been associated with optic ataxia - an impairment of reaching movements directed to visual targets (Perenin \& Vignetto, 1988; but see also Karnath \& Perenin, 2005). While optic ataxia has typically been described as an high-level impairment of reaching movements that results in a deficit in executing movements to visual targets, we have recently argued that the most common form of optic ataxia - so-called non-foveal optic ataxia (Buxbaum \& Coslett, 1997) - is in fact best explained as an impairment in the ability to selectively de-couple reach direction from gaze direction, i.e., to reach to a location away from the current point of fixation (Jackson et al., 2005). In our view, this deficit arises largely due to an inability to execute a movement that must be planned and controlled primarily in intrinsic (postural) coordinates. In this context it is noteworthy that a very recent neuropsychological study, which utilised MRI-based lesion reconstruction analyses to investigate the neural bases of optic ataxia in humans (Karnath \& Perenin, 2005), identified damage to the precuneus to be highly associated with optic ataxia. In this study Karnath and Perenin reconstructed the lesions of 10 individuals presenting with optic ataxia following unilateral left hemisphere lesions and six individuals presenting with optic ataxia following unilateral right hemisphere lesions. In both cases lesion overlays were contrasted with an appropriate group of controls who themselves presented with unilateral left or right hemisphere lesions but who did not exhibit optic ataxia. The resultant difference images revealed that, for both the right hemisphere and left hemisphere groups, the precuneus was damaged in greater than $60 \%$ of the individuals who presented with optic ataxia.

We note that the brain regions activated in our studies of reaching without vision to posturally defined target locations, correspond closely to a region of the PPC recently identified by Connolly et al. (2003) as the human homologue of the Parietal Reach Region (PRR) observed in the monkey brain. This finding is of particular interest as the PRR in the monkey has been widely reported to represent reaching movements in eyecentred coordinates (e.g., Batista et al., 1999), and this might be seen as a potential difficulty in light of our interpretation of the results from our study. In their fMRI study, Connolly et al. identified the medial aspect of the human superior parietal lobule as the homologue of the PRR based upon the fact that this region responded more strongly during a delay period when the individual was preparing to make a reaching movement compared to when they were preparing to make a saccade. However, two points are worth noting in relation to this finding. First, this area showed a substantial increase in bold response for both saccadic and reaching responses suggesting that this region may indeed respond to changes in eye position (see Battaglia-Mayer et al., in press). Second, these authors never demonstrate in their study that the medial superior parietal area identified at the human homologue of the PRR in fact codes reaching movements in eye-based coordinates (in many ways the defining aspect of the PRR in the monkey). Based upon these considerations, it strikes us that our study is consistent with the findings of Connolly et al. - confirming that the medial aspect of the superior parietal lobule may be important for the spatial coding of reaching movements - but also extends their finding by clearly demonstrating that this region is activated for movements made without vision to postural targets.

One question that is clearly not addressed by our current study is whether information that may be used to update spatial representations of the body posture arises from somatosensory processing areas coding proprioceptive inflow, from motor regions that may send an 'efference copy' of the motor outflow (thereby permitting the predicted 'next-state' of the body schema to be rapidly predicted (Wolpert \& Ghahramani, 2000)), or from a combination of both of these sources. This is an extremely important question that must await further investigation using other neuroimaging techniques.

\section{References}

Andersen, R. A., Snyder, L. H., Bradley, D. C., \& Xing, J. (1997). Annual Review Neuroscience, 20, 303-330.

Balint, R. (1909). Seelenla“hmung des 'schauens', optische ataxie, raumliche storung der aufmerksamkeit. Monattsschrifte fur Psychiatrische Neurologie, 25, 51-81.

Batista, A. P., Buneo, C. A., Snyder, L. H., \& Andersen, R. A. (1999). Reach plans in eye-centred coordinates. Science, 285, 257-260.

Battaglia-Mayer, A., Archambault, P. S., \& Caminiti, R. (in press). The cortical network for eye-hand coordination and its relevance to understanding motor disorders of parietal patients. Neuropsychologia, doi:10.1016/j.neuropsychologia.2005.11.021.

Battaglia-Mayer, A., \& Caminiti, R. (2002). Optic ataxia as a result of the breakdown of the global tuning fields of parietal neurones. Brain, 125, 225-237.

Beckmann, C. F., Jenkinson, M., \& Smith, S. M. (2003). General multilevel linear modelling for group analysis in FMRI. NeuroImage, 20, 1052-1063.

Buneo, C. A., \& Andersen, R. A. (in press). The posterior parietal cortex: Sensorimotor interface for the planning and online control of visually- 
guided movements. Neuropsychologia, doi:10.1016/j.neuropsychologia. 2005.10.011.

Buxbaum, L. J., \& Coslett, H. B. (1997). Subtypes of optic ataxia: Reframing the disconnection account. Neurocase, 3, 159-166.

Connolly, J. D., Andersen, R. A., \& Goodale, M. A. (2003). FMRI evidence for a 'parietal reach region' in the human brain. Experimental Brain Research, 153, 140-145.

Critchley, M. (1953). The Parietal Lobes. London: Edward Arnold.

De Renzi, E. (1996). Balint-Holmes syndrome. In C. Code, C.-W. Wallesch, Y. Joanette, \& A. Roch Lecours (Eds.), Classic Cases in Neuropsychology (pp. 123-143). Hove, UK: Psychology Press.

Gazzaniga, M. S. (2000). Brain, 123, 1293-1326.

Gross, C. G., \& Graziano, M. S. A. (1995). Multiple representations of space in the brain. The Neuroscientist, 1, 43-50.

Head, H., \& Holmes, G. (1911-1912). Sensory disturbances from cerebral lesions. Brain, 34, 102-254.

Jackson, S. R., Newport, R., Husain, M., Harvey, M., \& Hindle, J. V. (2000). Neuropsychologia, 38, 500-507.

Jackson, S. R., Newport, R., Mort, D., \& Husain, M. (2005). Where the eye looks, the hand follows: Limb-dependent magnetic misreaching in optic ataxia. Current Biology, 15, 42-46.

Jenkinson, M., Bannister, P., Brady, M., \& Smith, S. (2002). Improved optimization for the robust and accurate linear registration and motion correction of brain images. Neuroimage, 17, 825-841.

Karnath, H.-O., \& Perenin, M.-T. (2005). Cortical control of visually guided reaching: Evidence from patients with optic ataxia. Cerebral Cortex, 5, 1561-1569.

Liepmann, H., \& Maas, O. (1907). Fall von linksseitiger agraphie und apraxis bei rechtsseitiger lahmung. Journal Fur Psychologie Und Neurologie, 10, 214-227.

Mechelli, A., Henson, R. N., Price, C. J., \& Friston, K. J. (2003). Comparing event-related and epoch analysis in blocked design fMRI. NeuroImage, $18,806-810$

Perenin, M. T., \& Vighetto, A. (1988). Optic ataxia: A specific disruption in visuomotor mechanisms. Different aspects of the deficit in reaching for objects. Brain, 111, 643-674.
Ratcliffe, G., \& Davies-Jones, G. A. B. (1972). Defective visual localisation in focal brain wounds. Brain, 95, 49-60.

Rizzolatti, G., Fogassi, L., \& Gallese, V. (1997). Parietal cortex: From sight to action. Current Opinion in Neurobiology, 7, 562-567.

Rushworth, M. F. S., Nixon, P. D., \& Passingham, R. E. (1997). Parietal cortex and movement. 1. Movement selection and reaching. Experimental Brain Research, 117, 292-310.

Rushworth, M. F. S., Johansen-Berg, H., \& Young, S. A. (1998). Parietal cortex and spatial-postural transformation during arm movements. Journal of Neurophysiology, 79, 478-482.

Sakata, H., Takaoka, Y., Kawarasaki, A., \& Shibutani, H. (1973). Somatosensory properties of neurons in superior parietal cortex (area 5) of the rhesus monkey. Brain Research, 64, 85-102.

Sirigu, A., Cohen, L., Duhamel, J. R., Pillon, B., Dubois, B., \& Agid, Y. (1995). A selective impairment of hand posture for object utilization in apraxia. Cortex, 31, 41-56.

Sirigu, A., Duhamel, J. R., Cohen, L., Pillon, B., Dubois, B., \& Agid, Y. (1996). The mental representation of hand movements after parietal cortex damage. Science, 273, 1564-1568.

Snyder, L. H. (2000). Coordinate transformations for eye and arm. Current Opinion in Neurobiology, 10, 747-754.

Tzourio-Mazoyer, N., Landeau, B., Papathanassiou, D., Crivello, F., Etard, O., Delcroix, N., et al. (2002). Automated anatomical labeling of activations in SPM using a macroscopic anatomical parcellation of the MNI MRI single-subject brain. Neuroimage, 15, 273-289.

Wolpert, D. M., \& Ghahramani, Z. (2000). Computational principles of movement neuroscience. Nature Neuroscience, 3, 1212-1217.

Wolpert, D. M., Goodbody, S. J., \& Husain, M. (1998). Maintaining internal representations: The role of the human superior parietal lobe. Nature Neuroscience, 1, 529-533.

Woolrich, M. W., Ripley, B. D., Brady, M., \& Smith, S. M. (2001). Temporal autocorrelation in univariate linear modeling of FMRI data. Neuroimage, 14, 1370-1386.

Worsley, K. J., Evans, A. C., Marrett, S., \& Neelin, P. (1992). A threedimensional statistical analysis for CBF activation studies in human brain Journal of Cerebral Blood Flow and Metabolism, 12, 900-918. 